\title{
Planktonic Pantoum
}

Calanus copepods molt their opal teeth

Lost like fecal pellets from euphotic waters.

Tooth fairy nickels flung in the sediment,

Pelagic grazers munch spiny diatoms.

Lost like fecal pellets from euphotic waters,

Glassy snowballs plunge to the ocean floor.

Pelagic grazers munch spiny diatoms

Deep beyond photosynthetic light.

Glassy snowballs plunge to the ocean floor

Made of frustules fragile as dreams.

Deep beyond photosynthetic light,

Calanus copepods elude hungry fish. 